\title{
Relationship between Muscle Strength, Muscle Mass and BMD in Postmenopausal Female of Zhuang in Guangxi Province of China
}

\author{
Peizhao Shi1,2, Songyi Mo1, Jiahui Li'1, Qingxiu Feng1,3, Yuanxin Huang4, Xinling Ma5, Biao Li', \\ Ziyang Fang1, Huiling Liu' ${ }^{1}$, Guangwu Huang1, Ling Tang1 ${ }^{1}$ Jinhua Wang1,6* Xiaoyan Fang ${ }^{*}$ \\ ${ }^{1}$ School of Basic Medical Sciences, Youjiang Medical University for Nationalities, Baise, China \\ ${ }^{2}$ Department of Orthopedics, Affiliated Hospital of Hebei University of Engineering, Handan, China \\ ${ }^{3}$ Department of Clinical Medicine, Medical School of Nanjing University, Nanjing, China \\ ${ }^{4}$ Affiliated Hospital of Youjiang Medical University for Nationalities, Baise, China \\ ${ }^{5}$ School of Nursing, Youjiang Medical University for Nationalities, Baise, China \\ ${ }^{6}$ West China School of Basic Medical Sciences \& Forensic Medicine, Sichuan University, Chengdu, China \\ Email: spz5930821@126.com, ^00433@ymun.edu.cn, *wangjinhua@ymun.edu.cn
}

How to cite this paper: Shi, P.Z., Mo, S.Y., Li, J.H., Feng, Q.X., Huang, Y.X., Ma, X.L., Li, B., Fang, Z.Y., Liu, H.L., Huang, G.W., Tang, L., Wang, J.H. and Fang, X.Y. (2022) Relationship between Muscle Strength, Muscle Mass and BMD in Postmenopausal Female of Zhuang in Guangxi Province of China. Journal of Biosciences and Medicines, 10, 87-97.

https://doi.org/10.4236/jbm.2022.102009

Received: January 8, 2022

Accepted: February 11, 2022

Published: February 14, 2022

Copyright $\odot 2022$ by author(s) and Scientific Research Publishing Inc. This work is licensed under the Creative Commons Attribution International License (CC BY 4.0).

http://creativecommons.org/licenses/by/4.0/

cc) (i) Open Access

\begin{abstract}
Objective: To explore the correlation between muscle strength, muscle mass and bone mineral density (BMD) in Zhuang female population, body composition analysis and grip strength, and to analyze the possible influencing factors of BMD. Methods: 182 postmenopausal women were selected from $\mathrm{Gu}-$ angxi Province of China. Broadband ultrasound attenuation (BUA) was used to evaluate BMD. Grip dynamometer to assess muscle strength. Height, weight and muscle mass of each part were measured by body composition measuring instrument. Body mass index (BMI), skeletal muscle mass index (SMI) and limb skeletal muscle mass (SM) were calculated according to the measurement results. Results: BUA, grip strength and SMI in postmenopausal women of Zhuang nationality showed a decreasing trend with age $(p<0.001)$. After normalization ( $\mathrm{Z}$ value/SD), BUA and SMI decay at a similar rate $(p<$ $0.001)$, and both decay more rapidly than grip strength $(p<0.001)$. After adjusting for age, partial correlation analysis showed that there was a high correlation between SM and BUA $(r=0.305, p<0.001)$ Multiple linear regression analysis showed that lower limb muscle mass (LSM) and age were independent factors of BUA $\left(\mathrm{R}^{2}=0.350\right)$. Only when age was not considered, the combination of grip strength and LSM was the optimal solution to reflect the calcaneal bone density of Zhuang women $\left(\mathrm{R}^{2}=0.306\right)$. Conclusion: With the increase of age, the decline rate of muscle strength of postmenopausal Zhuang women in Guangxi is slower than that of BMD and muscle mass. SM can better reflect the BMD level of the body than SMI, and the LSM is the main
\end{abstract}


influencing factor of BMD.

\section{Keywords}

Muscle Strength, Muscle Mass, Musculoskeletal System, Bone Mineral

Density, Postmenopausal Females

\section{Introduction}

Osteoporosis (OP) is a kind of systemic bone disease characterized by bone mass loss, damage to fine bone structure and brittle fracture (World Health Organization, WHO). Middle-aged and elderly females due to the accelerated bone loss caused by decreased postmenopausal estrogen levels caused by osteoporosis, called postmenopausal osteoporosis (postmenopausal osteoporosis, PMO) [1]. Related studies show that PMO has become an important health problem for females over 50 years old in China [2]. Epidemiological survey shows that the prevalence rate of OP in Chinese people over 50 years old is $14.4 \%$ for men and $20.7 \%$ for females, and the prevalence difference over 60 years old is more obvious [3].

Double-energy X-ray (DXA) bone density (BMD) examination is considered the "standard" for early-stage examination and diagnosis of OP. Quantitative ultrasound BMD examination technique, mainly calcaneus broadband ultrasound attenuation (BUA), evaluates the BMD by examining the ultrasonic amplitude of different bone mass and frequency of bone tissue. OP screening is an important measure for its prevention and control and clinical diagnosis and treatment. At present, BMD screening technology has corresponded defects. Although BUA is questioning the reliability of OP diagnosis than DXA, it is still considered a simple, economical and practical technology from the perspective of OP screening effectiveness of epidemiological research [4].

Skeletal muscle and skeleton are the basic structures for human beings to realize life activities. The structure and function of musculoskeletal is interdependent and crosstalk. More and more attention has been paid to the correlation between muscle mass, strength and bone mineral density (BMD) [5]. A positive correlation between muscle mass and BMD has been shown [6]. Low muscle mass and grip strength can predict the risk of brittle fracture [7]. Like BMD, muscle mass and strength in menopausal females may decline at all anatomical levels as estrogen levels decline, and individual menopausal status is an important predictor of muscle mass and function. It may be influenced by many factors such as nationality, region, cultural customs, economy and lifestyle [8]. In this study, postmenopausal females in regional villages and towns were selected to explore the changes of muscle strength, muscle mass and BMD with age after menopause, to study the correlation between muscle strength, limb muscle mass (SM) and calcaneal BMD, and to analyze the possible factors affecting calcaneal BMD. 


\section{Materials and Methods}

\subsection{Subjects}

This is a cross-sectional study performed in Guangxi Province of China. Postmenopausal women of Zhuang nationality of China were included via the random cluster sampling from four counties (Debao, Tiandeng, Daxin, and Wumin) and one city (Yizhou) of Guangxi Province. The inclusion criteria were: 1) healthy women aged 45 years or above; 2) of Zhuang nationality for at least of three generations; and 3) after natural menopausing for at least five years. Women with either of the following clinical conditions were excluded: 1) with concurrent medications that may affect skeletal muscle or bone metabolism; 2) patients with any chronic illnesses, recent trauma, acute diseases, or surgeries; 3) with psychological stress or conditions that may affect physical health, especially those with abnormal muscle and bone metabolism caused by abnormal menstrual cycle and early menopause. 182 eligible subjects were included in the study. The protocol of the study was approved by the Youjiang Medical University for Nationalities, and all of the included participants signed the consents before they were enrolled into this study.

\subsection{Anthropometrics}

Weight was measured (with shoes and bulky clothing removed) by using a single pair of electronic scales which was calibrated using a known weight at the beginning. Height was measured by using a stadiometer. Body mass index (BMI) was calculated [weight $(\mathrm{kg}) /$ height $(\mathrm{m})^{2}$ ]. Bioelectrical impedance analyzer (TANITA, MC-180, Japan) was used to measure the lean body weight (LBW), body fat rate (BF\%), muscle mass of upper, lower limbs and trunk, and the unit of measurement was kilogram. All fours skeletal muscle mass (ASM) is the sum of all fours skeletal muscle mass, measured in kilograms. Skeletal muscle mass index (skeletal muscle mass index, SMI) is ASM divided by the square of height, measured in kilograms per square meter $\left(\mathrm{kg} / \mathrm{m}^{2}\right)$.

\subsection{BUA Measurement}

The calcaneus BUA was selected to evaluate the BMD level according to relevant studies [9]. The subject's right calcaneus BUA value $(\mathrm{dB} / \mathrm{MHz})$ was measured using a calcaneus ultrasound bone densitometer (OSTEOSPACE, France). Standard calibration was performed before each measurement, and the operation was performed by fixed professionals.

\subsection{Muscle Strength Measurement}

The muscle strength of the subject was assessed using the grip test: WCS-10000 grip meter (Wanqing Electronics, Shanghai) in unit of kg. Before testing the grip strength was adjusted within the appropriate range, the subject's test arm was slightly extended, but not more than $30^{\circ}$, the upper arm and forearm sat at $90^{\circ}$ seated, pulled the handle of the instrument with maximum strength, held both 
hands two times, and took the maximum value for statistical analysis.

\subsection{Statistical Analysis}

The data of normal distribution was expressed as mean \pm standard deviation, and a comparison between multiple groups was made by using Welch's ANOVA. The linear relationship, Pearson's correlation and multiple logistic regression were used. $p$ value $<0.05$ (two-tailed) or a 95\% confidence interval (CI) not including the null point was regarded as statistically significant. All statistical analyses were performed using SPSS 26.0 for Windows (SPSS, Chicago, IL, USA).

\section{Results}

182 Zhuang female subjects average age was $60.61 \pm 8.94$ years (Table 1 ). The average height was $149.47 \pm 6.26 \mathrm{~cm}$, and the average body weight was $52.43 \pm$ $8.60 \mathrm{~kg}$. Height $(p<0.001)$, body weight $(p=0.02)$ and LBW $(p<0.001)$ decreased with age. Grip strength, BUA, muscle strength, upper limb muscle mass, lower limb muscle mass, SM and SMI varied between groups among age groups, generally associated with increased age and showed a downward trend (Table 1). However, the BMI, BF\% and the trunk muscle mass showed no statistical difference between the age groups $(p>0.05)$.

Table 1. Characteristics of participants (split by age group).

\begin{tabular}{|c|c|c|c|c|c|c|}
\hline \multirow{2}{*}{ Subjects, $\mathrm{n}$} & Total & $45-54$ & $55-64$ & $65-74$ & $75+$ & $p$ value \\
\hline & 182 & 53 & 70 & 44 & 15 & \\
\hline Age, yrs & $60.61 \pm 8.94$ & $50.23 \pm 2.93$ & $59.46 \pm 2.57^{* *}$ & $69.61 \pm 3.34^{* *}$ & $76.27 \pm 1.39^{* *}$ & $<0.001$ \\
\hline Height, $\mathrm{cm}$ & $149.47 \pm 6.26$ & $151.71 \pm 5.15$ & $150.26 \pm 5.55$ & $147.72 \pm 6.20$ & $143.12 \pm 7.98$ & $<0.001$ \\
\hline Weight, kg & $52.43 \pm 8.60$ & $54.50 \pm 7.90$ & $53.29 \pm 7.99$ & $50.08 \pm 9.53$ & $48.05 \pm 8.51$ & 0.020 \\
\hline LBW, kg & $36.78 \pm 3.70$ & $38.59 \pm 2.97$ & $37.12 \pm 3.05^{*}$ & $35.04 \pm 3.74$ & $33.88 \pm 4.91$ & $<0.001$ \\
\hline $\mathrm{BF} \%, \%$ & $28.95 \pm 6.91$ & $28.37 \pm 6.74$ & $29.53 \pm 6.63$ & $28.78 \pm 8.03$ & $28.81 \pm 5.57$ & 0.828 \\
\hline BMI, kg/m² & $23.38 \pm 3.07$ & $23.61 \pm 3.05$ & $23.57 \pm 2.85$ & $22.85 \pm 3.57$ & $23.27 \pm 2.67$ & 0.673 \\
\hline Grip Strength, kg & $17.55 \pm 5.70$ & $20.12 \pm 5.25$ & $18.12 \pm 5.00$ & $15.16 \pm 5.99^{*}$ & $13.25 \pm 4.47$ & $<0.001$ \\
\hline BUA, dB/MHz & $54.17 \pm 7.50$ & $60.11 \pm 6.88$ & $53.46 \pm 5.89^{* *}$ & $50.35 \pm 6.17^{* *}$ & $47.64 \pm 6.00$ & $<0.001$ \\
\hline \multicolumn{7}{|l|}{ Muscle Mass, kg } \\
\hline Total body & $34.72 \pm 3.38$ & $36.37 \pm 2.71$ & $35.03 \pm 2.80^{* *}$ & $33.13 \pm 3.41^{* *}$ & $32.08 \pm 4.48$ & $<0.001$ \\
\hline Trunk & $19.73 \pm 1.81$ & $19.90 \pm 1.41$ & $19.96 \pm 1.79$ & $19.21 \pm 1.75$ & $19.57 \pm 2.91$ & 0.146 \\
\hline Upper Limb & $3.43 \pm 0.62$ & $3.62 \pm 0.46$ & $3.45 \pm 0.39$ & $3.32 \pm 0.89$ & $3.05 \pm 0.83$ & 0.020 \\
\hline Lower Limb & $11.66 \pm 1.57$ & $12.94 \pm 1.22$ & $11.72 \pm 1.01^{* *}$ & $10.71 \pm 1.38^{* *}$ & $9.58 \pm 1.33^{* *}$ & $<0.001$ \\
\hline SM, kg & $15.09 \pm 2.01$ & $16.56 \pm 1.63$ & $15.17 \pm 1.33^{*}$ & $14.03 \pm 1.97^{* *}$ & $12.63 \pm 1.97$ & $<0.001$ \\
\hline SMI, kg/m² & $6.74 \pm 0.66$ & $7.19 \pm 0.59$ & $6.72 \pm 0.48^{*}$ & $6.42 \pm 0.69$ & $6.12 \pm 0.49$ & $<0.001$ \\
\hline
\end{tabular}

${ }^{*}$ Compared with the previous age group, it was statistically significant $(p<0.05),{ }^{* *} P<0.01$. Data in shaded cells statistically significant results. LBW: Lean Body Weight. BF\%: Body Fat Rate. BMI: Body Mass Index. BUA: Broadband Ultrasound Attenuation. SM: Skeletal Muscle Mass. SMI: Skeletal Muscle Mass Index. 
The grip strength, SMI and calcaneal BUA were converted to the corresponding $\mathrm{Z}$ values for simple linear regression analysis. As shown in Figure 1(a) and Figure $1(\mathrm{~b})$, the $\mathrm{Z}$ values of grip strength and SMI decreased with age $(p<$ $0.001)$. When the age increased by 10 years, the grip strength and SMI decreased by $0.4 \mathrm{SD}$ and $0.6 \mathrm{SD}$, respectively. As shown in Figure 1(c), the BUA decreased with age $(p<0.001)$, and the BUA decreased by 0.6 SD for every 10 years of age.Normalized BUA decreased more rapidly with age than grip strength $(p<$ $0.001)$, it was not significantly different from SMI $(p=0.713)$.

Partial correlation analysis after adjusted age showed that grip strength, SMI, muscle mass, muscle mass of each part and ASM were positively correlated with BUA of calcaneus (Table 2). Among them, ASM $(r=0.305, p<0.001)$, lower limb muscle mass $(r=0.302, p<0.001)$ have strong correlation with BUA. However, grip strength $(r=0.138, p=0.032)$, trunk muscle mass $(r=0.163, p=$ $0.014)$ are weakly correlated with BUA.

Table 2. Correlation analysis of grip strength, SMI, ASM, muscle strength in various parts and calcaneal BUA after adjusted age.

\begin{tabular}{ccc}
\hline & \multicolumn{2}{c}{ BUA } \\
\cline { 2 - 3 } & $r$ & $p$ \\
\hline Grip Strength & 0.138 & 0.032 \\
\hline SMI & 0.234 & 0.001 \\
SM & 0.305 & $<0.001$ \\
\hline Muscle Mass & & \\
\hline Upper Limb & 0.231 & 0.001 \\
Lower Limb & 0.302 & $<0.001$ \\
Trunk & 0.163 & 0.014
\end{tabular}

SM: Skeletal Muscle Mass. SMI: Skeletal Muscle Mass Index.
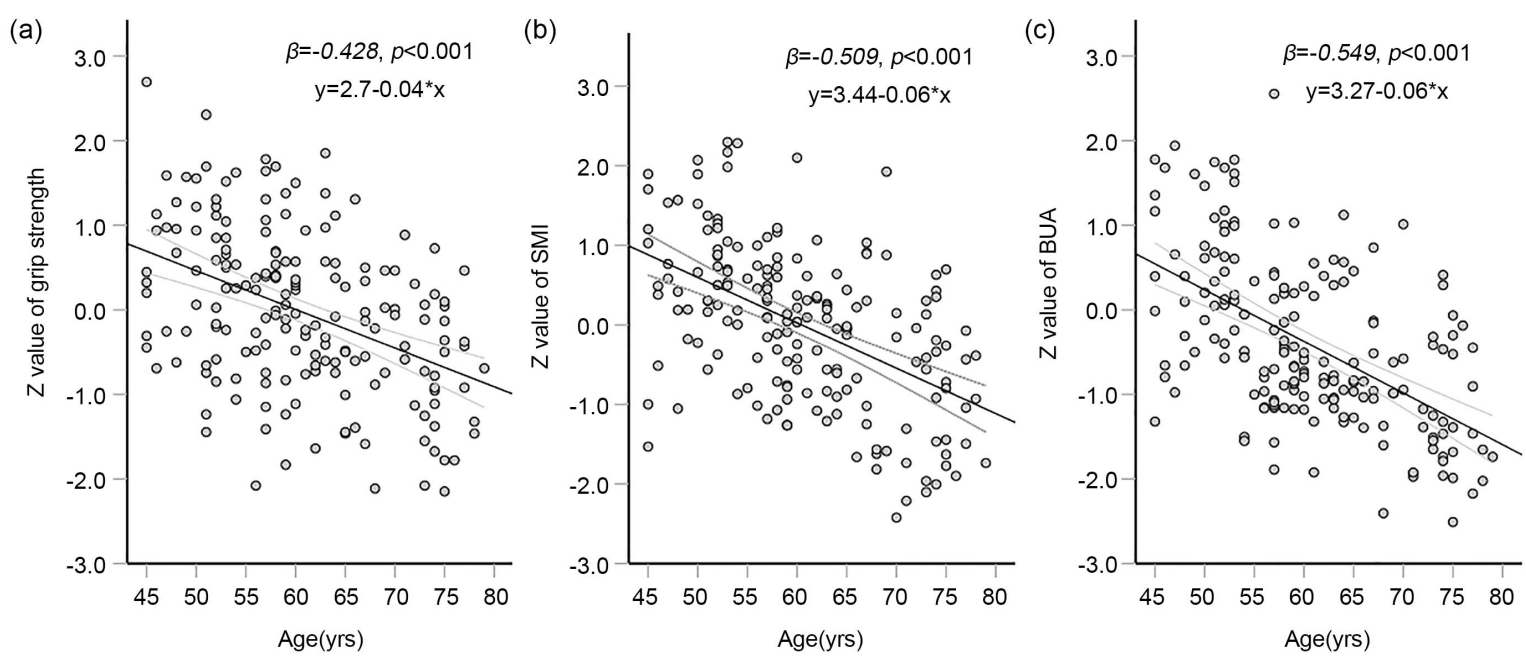

Figure 1. Scatter plot for associations between age and grip strength, SMI and BUA with multivariable analyses. The Z value $((X-\mu) / \sigma)$ of grip strength (a), SMI (b) and BUA (c) showed an inverse relationship with age. BUA: Broadband Ultrasound Attenuation. SMI: Skeletal Muscle Mass Index. 
The results in Table 3 show that there is a positive correlation between lower limb muscle mass and BUA, and it can be used as an index representing muscle mass to independently evaluate BUA level (Table 3, Model 1). Without considering the influence of age, grip strength can be used together with lower limb muscle strength to evaluate calcaneal bone mineral density (Table 3, Model 2). As the grip strength is greatly influenced by age. When the age variable is included, age can replace grip strength and lower limb muscle mass to evaluate BUA (Table 3, Model 3).

\section{Discussion}

This study shows that the change of BMD of the calcaneus of postmenopausal females of Zhuang is faster than that of muscle strength, and there are significant differences in many indexes $(p<0.01)$. It is considered that with the increase of age, the strength of human bones gradually decreases and the brittleness gradually increases, until it is not enough to resist the external force and brittle fracture occurs, which has a serious impact on the quality of life and natural life of the elderly [1]. After menopause, females' aging is accelerated, and bone loss is accelerated. Bone loss can occur in both cancellous bone and cortical bone, among which cancellous bone loss shows sparse bone texture. The loss of cortical bone is accompanied by the decrease of bone mass and bone strength, which together reduce the bone density [10]. Because of the unique physiological change of menopause, the prevalence of osteoporosis in middle-aged and elderly females is much higher than that in males [2]. More than $90 \%$ of limb bone mass loss occurs during and after menopause, and more than $80 \%$ is cortical bone, which may explain that $80 \%$ of fractures are the causes of limb fractures [11]. The BMD of the calcaneus of postmenopausal females of Zhuang nationality tends to decline with age (Figure 1), and its regularity is consistent with these reports. Research on the correlation between muscle mass, strength and bone mass shows that muscle strength is positively correlated with BMD, but there was no significant correlation with muscle mass [12]. This is related to the decrease of sensitivity of osteoblast dendrites to mechanical stimulation caused by muscle contraction due to the decrease of estrogen [13]. In our study, the grip strength of the postmenopausal females of Zhuang nationality is positively correlated with BUA, it is not significantly correlated with SMI, and the bone density declines faster than the muscle strength. Related reports show that muscle strength is related to age, fat mass and BMI. Multi-factor research and observation are needed [14].

The muscle mass and muscle strength of postmenopausal Zhuang females show a declining and accelerating trend with the increase of age. The statistical results of this paper show that the muscle mass decays faster than the muscle strength. Existing data shows that the difference between different evaluation criteria of muscle quality and muscle strength mainly lies in different evaluation indexes and different critical values. At present, most evaluation systems use 
Table 3. Optimal solutions of multiple linear regression based on calcaneal BUA.

\begin{tabular}{ccccc}
\hline & \multicolumn{2}{c}{ BUA } & \multirow{2}{*}{$\mathrm{R}^{2}$} & residual \\
\cline { 2 - 3 } & \multicolumn{1}{c}{$\boldsymbol{\beta}$} & $\boldsymbol{p}$ & & \\
\hline Model 1 & & & & \\
\hline Lower Limb Muscle Mass & 0.546 & $<0.0001$ & 0.298 & 7150.92 \\
\hline Model 2 $^{1)}$ & & & & \\
\hline Lower Limb Muscle Mass & 0.495 & $<0.0001$ & \multirow{2}{*}{0.306} & 6991.12 \\
Grip Strength & 0.135 & 0.045 & & \\
\hline Model 3 & & & & \\
\hline Lower Limb Muscle Mass & 0.336 & $<0.0001$ & & \\
Age & -0.322 & $<0.0001$ & & \\
\hline
\end{tabular}

1) Optimal solution without considering the effect of age. 2) Optimal solution considering the effect of age.

muscle quality and/or muscle strength as the main evaluation indexes. It is considered that the aging of muscle is related to the decline of muscle strength and muscle mass [14]. Studies have shown that the relationship between muscle strength and muscle mass is not linear, and this nonlinear relationship is related to the degree of local or systemic aseptic inflammation [15]. PMO is definitely caused by the aging of musculoskeletal cells induced by estrogen decline and the steady-state imbalance of inflammatory response, which leads to local or systemic inflammation leading to the imbalance of musculoskeletal remodeling [16]. Myoskeletal pain, especially chronic musculoskeletal pain, induced by the stimulation of musculoskeletal inflammation can affect physical activities, especially exercise, leading to the decrease of muscle strength. If musculoskeletal pain cannot be alleviated or eliminated, lack of continuous exercise leads to a decline in muscle mass. In this paper, there are significant differences in muscle mass and strength among female workers with different physical strength in the same group, which may affect the differences in muscle mass and strength among different age groups. Mechanical stimulation generated by muscle activities of different intensities directly regulates the metabolism of muscle protein or immune factors. And it is related to individual nutrition, psychology and environmental factors. Single exercise intensity has no clear correlation with muscle quality or strength, but is directly related to age and sex hormone decline, which is the mechanism of differences among different age groups [8].

SMI, as the most commonly used indicator of muscle mass, is highly correlated with BMD in different population studies [15] [17] [18]. In our study, the correlation between SM and calcaneal BMD is stronger than SMI. The reason may be that SMI removes the influence of height, but there is a correlation between height and bone mineral density [13] [19]. It is generally believed that the height of adults is more constant than the weight. In the process of studying myopathy, the influence of different body shapes can be eliminated to the great- 
est extent by adjusting the height. However, in the research of analyzing the correlation between postmenopausal females and BMD, the rapid decline of BMD, the loss of trabecular bone in the spine and the decrease of the integrity of the three-dimensional structure led to the significant reduction of the weight-bearing capacity of the vertebral body. Furthermore, the collapse and morphological changes of vertebral body leads to the decrease of the overall height of the spine, while the simultaneous pathological curvature of the spine aggravates the decrease of the overall height, which in turn leads to the overall decrease of the height. In this study, the correlation between the height of subjects and BUA of calcaneus was statistically significant $(r=0.160, p=0.031)$, which was the main reason that the correlation between SM and BMD of calcaneus was stronger than SMI.

There are also differences in the correlation between BMD in different parts of an individual and muscle quality and strength in different parts [20]. However, BMD of the same part has the strongest correlation with muscle mass [21]. Muscle imaging is a common technique for evaluating muscle mass and bone density at present. Digital X-ray, CT, MRI and quantitative ultrasound (QUS) have their own advantages and disadvantages. Among them, measurement of calcaneal BUA by QUS combined with analysis of human composition is an independent correlation technique for predicting fragile fractures of femoral neck, distal radius and lumbar spine [22]. In our study, the correlation between lower limb muscle mass and BUA $(r=0.302)$ is second only to limb SM. In the regression model constructed by step-by-step method (Table 3, Model 3), lower limb muscle mass $(\beta=0.336)$ and age $(\beta=-0.322)$ constitute the optimal solution. This result shows that the lower limb muscle mass is an independent and age-dependent influencing factor of calcaneal BMD. When age is not included in the regression model (Table 3, Model 2). At the same time, females with higher grip strength and lower limb muscle mass have higher BMD, among which the lower limb muscle mass has greater influence on bone mineral density than muscle strength, and its mechanism is related to the crosstalk between musculoskeletal.

\section{Limitations of This Study}

In our study, the selection of subjects, factors such as regional cultural economy, customs and habits, past and current diseases that may affect musculoskeletal metabolism were considered, which made the sample size less. Considering the practicability and convenience of on-site screening equipment technology in villages and towns. Moreover, it can reduce the economic burden and choose simple and practical BUA, bioelectric body composition analysis and grip measurement inspection technology, which is different from the scientificity and reliability of the current OP inspection mainstream technology. Muscle strength test and bone density test in different parts should be added to better evaluate the relationship between muscle and bone. We will further investigate the influence of different nationalities, different lifestyles and customs on musculoskeletal de- 
generation, especially the pathophysiology of $\mathrm{PMO}$, and provide reference for regional PMO prevention and control.

\section{Conclusion}

The calcaneal BUA, muscle mass and the decline rate with age were faster than the muscle strength in postmenopausal women of Zhuang nationality in Guangxi. SM can reflect the BMD level better than SMI, and the lower limb muscle mass is the main influencing factor of calcaneal BMD. Compared with muscle mass, muscle strength was less affected by menopause, and the effect of muscle strength on bone mineral density was weakened by menopause. It is more meaningful for us to use SM instead of SMI to study the correlation between muscle mass and bone mineral density in future studies.

\section{Funding}

Project supported by Academic Degree Graduate Innovation Program of Youjiang Medical University for Nationalities 2020 (Project No. YXCXJH2020004)

\section{Conflicts of Interest}

The authors declare no conflicts of interest regarding the publication of this paper.

\section{References}

[1] Kanis, J.A., Cooper, C., Rizzoli, R. and Reginster, J.Y. (2019) European Guidance for the Diagnosis and Management of Osteoporosis in Postmenopausal Women. Osteoporosis International, 30, 3-44. https://doi.org/10.1007/s00198-018-4704-5

[2] Chinese Society of Osteoporosis and Bone Mineral Research (2019) Osteoporosis in China: An Epidemiological Survey and the Results of "Healthy Bones" Special Action. Chinese Journal of Osteoporosis and Bone Mineral Research, 12, 317-318.

[3] Xia, W., Zhang, Z., Lin, H., Jin, X., Yu, W. and Fu, Q. (2019) Guidelines for the Management of Primary Osteoporosis (2017). Chinese Journal of Osteoporosis, 25, 281-309.

[4] Xu, Y., Xu, Y., Ding, Z., Chen, Y., Su, B., Ma, Z., et al. (2017) Correlation between Ultrasonic Power Spectrum and Bone Density on the Heel. Ultrasonics, 73, 77-81. https://doi.org/10.1016/j.ultras.2016.08.023

[5] Sipilä, S., Törmäkangas, T., Sillanpää, E., Aukee, P., Kujala, U.M., Kovanen, V., et al. (2020) Muscle and Bone Mass in Middle-Aged Women: Role of Menopausal Status and Physical Activity. Journal of Cachexia, Sarcopenia and Muscle, 11, 698-709. https://doi.org/10.1002/jcsm.12547

[6] Kim, J.H., Hong, A.R., Choi, H.J., Ku, E.J., Lee, J.H., Cho, N.H., et al. (2018) Defining Sarcopenia in Terms of Skeletal Health. Archives of Osteoporosis, 13, Article No. 100. https://doi.org/10.1007/s11657-018-0511-z

[7] Juppi, H.K., Sipilä, S., Cronin, N.J., Karvinen, S., Karppinen, J.E., Tammelin, T.H., et al. (2020) Role of Menopausal Transition and Physical Activity in Loss of Lean and Muscle Mass: A Follow-Up Study in Middle-Aged Finnish Women. Journal of Clinical Medicine, 9, Article No. 1588. https://doi.org/10.3390/jcm9051588 
[8] Kult, S., Olender, T., Osterwalder, M., Markman, S., Leshkowitz, D., Krief, S., et al. (2021) Bi-Fated Tendon-to-Bone Attachment Cells Are Regulated by Shared Enhancers and KLF Transcription Factors. ELife, 10, Article ID: e55361. https://doi.org/10.7554/elife.55361

[9] Wang, J.-H., Huang, X.-F. and Zhou, Q.-H. (2012) Study on the Peak Bone Mass by Calcaneal Quantitative Ultrasound in Zhuang Healthy Women. Modern Preventive Medicine, 39, 838-840.

[10] Borgström, F., Karlsson, L., Ortsäter, G., Norton, N., Halbout, P., Cooper, C., et al. (2020) Fragility Fractures in Europe: Burden, Management and Opportunities. Archives of Osteoporosis, 15, Article No. 59. https://doi.org/10.1007/s11657-020-0706-y

[11] Armbrecht, G., Nguyen Minh, H., Massmann, J. and Raum, K (2021) Pore-Size Distribution and Frequency-Dependent Attenuation in Human Cortical Tibia Bone Discriminate Fragility Fractures in Postmenopausal Women with Low Bone Mineral Density. JBMR Plus, 5, Article ID: e10536. https://doi.org/10.1002/jbm4.10536

[12] Qiu, S., Divine, G., Warner, E. and Rao, S.D. (2020) Reference Intervals for Bone Histomorphometric Measurements Based on Data from Healthy Premenopausal Women. Calcified Tissue International, 107, 543-550.

[13] Tachiki, T., Kouda, K., Dongmei, N., Tamaki, J., Iki, M., Kitagawa, J., et al. (2019) Muscle Strength Is Associated with Bone Health Independently of Muscle Mass in Postmenopausal Women: The Japanese Population-Based Osteoporosis Study. Journal of Bone and Mineral Metabolism, 37, 53-59. https://doi.org/10.1007/s00774-017-0895-7

[14] Lewis, K.J., Cabahug-Zuckerman, P., Boorman-Padgett, J.F., Basta-Pljakic, J., Louie J., Stephen, S., et al. (2021) Estrogen Depletion on in Vivo Osteocyte Calcium Signaling Responses to Mechanical Loading. Bone, 152, Article ID: 116072. https://doi.org/10.1016/j.bone.2021.116072

[15] Da Cruz, G.F., Lunz, T.M., de Jesus, T.R., Costa, M.B., Vidigal, C.V., Albergaria, B.H., et al. (2021) Influence of the Appendicular Skeletal Muscle Mass Index on the Bone Mineral Density of Postmenopausal Women. BMC Musculoskeletal Disorders, 22, Article No. 861. https://doi.org/10.1186/s12891-021-04748-x

[16] Tuttle, C., Thang, L. and Maier, A.B. (2020) Markers of Inflammation and Their Association with Muscle Strength and Mass: A Systematic Review and Meta-Analysis. Ageing Research Reviews, 64, Article ID: 101185.

https://doi.org/10.1016/j.arr.2020.101185

[17] Hyde, N.K., Duckham, R.L., Wark, J.D., Brennan-Olsen, S.L., Hosking, S.M., Holloway-Kew, K.L., et al. (2020) The Association between Muscle Mass and Strength in Relation to Bone Measures in a Paediatric Population: Sex-Specific Effects. Calcified Tissue International, 107, 121-125. https://doi.org/10.1007/s00223-020-00699-y

[18] Erickson, K.R., Grosicki, G.J., Mercado, M. and Riemann, B.L. (2020) Bone Mineral Density and Muscle Mass in Masters Olympic Weightlifters and Runners. Journal of Aging and Physical Activity, 28, 749-755. https://doi.org/10.1123/japa.2019-0426

[19] Bohannon, R.W. (2019) Grip Strength: An Indispensable Biomarker for Older Adults. Clinical Interventions in Aging, 14, 1681-1691.

https://doi.org/10.2147/cia.s194543

[20] Vitale, J.A., Sansoni, V., Faraldi, M., Messina, C., Verdelli, C., Lombardi, G., et al. (2021) Circulating Carboxylated Osteocalcin Correlates with Skeletal Muscle Mass and Risk of Fall in Postmenopausal Osteoporotic Women. Frontiers in Endocrinology, 12, Article ID: 669704. https://doi.org/10.3389/fendo.2021.669704 
[21] Zengin, A., Pye, S.R., Cook, M.J., Adams, J.E., Rawer, R., Wu, F., et al. (2017) Associations of Muscle Force, Power, Cross-Sectional Muscle Area and Bone Geometry in Older UK Men. Journal of Cachexia, Sarcopenia and Muscle, 8, 598-606. https://doi.org/10.1002/jcsm.12198

[22] Guerri, S., Mercatelli, D., Aparisi, G.M., Napoli, A., Battista, G., Guglielmi, G., et al. (2018) Quantitative Imaging Techniques for the Assessment of Osteoporosis and Sarcopenia. Quantitative Imaging in Medicine and Surgery, 8, 60-85.

https://doi.org/10.21037/qims.2018.01.05 